\title{
A practical method for determining transmission usage-based tariff in electricity markets with different trading modes
}

\author{
Zhaoxia Jing, Xianzhong Duan, Member, IEEE, Yixin Ni, Senior Member, IEEE, Fushuan Wen, and \\ Felix F. Wu, Fellow, IEEE
}

\begin{abstract}
In this paper, a practical approach is presented for determining transmission usage-based tariff, based on the wellknown Generation Shift Distribution Factor (GSDF) based method and the recently developed power flow tracing method. First, the GSDF based method is employed for allocating the transmission usage-based charges among various transactions, and then the power flow tracing technique used for further distributing the charge such obtained among the participants of each transaction concerned. The proposed method is easy to implement and is applicable to electricity markets with various trading modes such as bilateral, multilateral and pool trading modes, and to interconnected multiple-regional and hierarchical electricity markets as well.
\end{abstract}

Index Terms--electricity market; transmission usage charge; transmission cost allocation; generation shift distribution factor (GSDF); power flow tracing method

\section{INTRODUCTION}

$\mathrm{A}$ $\mathrm{N}$ appropriate transmission pricing approach can promote economic use of present transmission resources and provide economic signals for future generation programming and load addressing, and is the foundation of good electricity market operation. In the implemented electricity markets, transmission variable costs and fixed costs are usually priced with different methods, that is, two-part tariff pricing method. For transmission variable costs, which are mainly composed of congestion costs and losses, the marginal-cost based spot pricing method can be used to provide economic signals; but for the fixed costs, since the marginal-cost based method cannot make the network owner break-even and is not appropriate, they are often covered through connection tariff and usage-based tariff. Usually connection tariff is determined by the connection points, the capacities of market participants (generators or loads) and other factors, but has no

This project is jointly supported by National Key Basic Research Special Fund of China (No. G1998020305), a seed funding project from the University of Hong Kong, and a specialized research fund for the doctoral program of higher education (SRFDP), China.

Z. X. Jing and X. Z. Duan are with the School of Electrical and Electronics Engineering, Huazhong University of Science \& Technology, Wuhan 430074 China (e-mail: jingzhaoxia@hotmail.com, xzduan2@263.net ).

F. S. Wen, Y. X. Ni and F.F. Wu are with the Department of Electrical and Electronic Engineering, The University of Hong Kong, Pokfulam Road, Hong Kong (e-mail: fswen@eee.hku.hk, yxni@eee.hku.hk, ffwu@eee.hku.hk). relation with actual network use of transmission users. On the other hand, usage-based tariff usually has strong relation with the actual network usage of transmission users. In this paper, the discussion will be restricted in the allocation of transmission usage-based charge.

As we know, the load flow function is a nonlinear function and superposition principle is not appropriate. Since different transactions interact with each other through voltages and branch currents, there is no theoretical exact method to determine the contribution of different market participants to network flow. Under different assumptions and simplifications, different methods, including DC load flow based method, are developed. Since DC load flow function is linear, superposition principle is appropriate and the calculation is simple and quick. Therefore, DC load flow based transmission fixed costs allocation methods are widely used in electricity markets.

The concept of GSDF (Generation Shift Distribution Factor) is used in many DC load flow based transmission fixed costs allocation methods ${ }^{[1-4]}$. In transmission costs allocation, GSDFs, which present the incremental change of network flows due to change of node injections, actually reflect the increment change of network use of generations and loads represented with node injections due to their change of injections and can be used to allocate transmission costs to generations and loads corresponding to node net injections. GSDFs cannot be affected by the variety of system operation conditions, and the GSDF based allocation method is easy to calculate, has clear physical concept and can provide correct economic signals. One of the main disadvantages of GSDF based allocation method is that the usage-based charges of market participants calculated by this method could be affected by the selection of reference node. The nearer to the load center the reference node locates, the higher percentage the generators will bear; the nearer to the generation center the reference node locates, the higher percentage the loads will bear. But for balanced bilateral or multilateral transactions, which mean that the sum of power injections in all related nodes is zero, their usage-based tariffs will not affected by the location of reference node. Of course, the transmission losses caused by the transactions should be dealt with separately. Thus, the GSDF based method can be used to calculate transmission usage-based tariff (TUT) for balanced 
transactions. Regarding to the transaction made up of all participants in pool as a large multilateral transaction, this method can also be applicable for the pool mode. Without additional declaration, the term "transaction" in this paper refers to balanced bilateral or multilateral transactions.

For bilateral transactions, it is $\mathrm{Ok}$ to allocate the transmission usage-based charge to transactions and the allocation of the charge among the transaction participants can be done by negotiation. But for multilateral transactions and pool transactions, it is not enough to allocate the charge to transactions, and the charge should further be allocated to different transaction participants. Due to the problem of reference selection discussed above, the GSDF based method is not appropriate for the allocation of transaction usage-based charges to different transaction participants.

The proportional-sharing-principle based power flow tracing method ${ }^{[5-7]}$ is a kind of physical-flow based power flow composition analysis method. In the application of this method, there will be no counter flow problem, which means that all participants should pay non-negative transmission charge, and the allocation result cannot be affected by the selection of reference node. On the other hand, this method cannot allocate transmission costs based on transactions. The GSDF based method and power flow tracing based method have different advantages and disadvantages and can remedy disadvantages each other. Therefore, this paper presented a practical method for determining Transmission Usage-based Tariff (TUT) synthetically by the two methods.First, GSDF based method is employed for allocating the transmission usage-based charges among various transactions, and then power flow tracing technique used for further distributing the charge such obtained among the participants of each transaction concerned. The proposed method is easy to implement and is applicable to electricity markets with various trading modes such as bilateral, multilateral and pool trading modes, and to interconnected multiple-regional and hierarchical electricity markets as well.

\section{ALLOCATION OF TUT AMONG ENERGY TRANSACTIONS}

In the proposed method, GSDF based method is firstly used to calculate the TUT of energy transactions.

For a network with $n$ nodes and $b$ branches, under DC assumptions, we could get

$$
P_{i j}=(\theta i-\theta j) / x_{i j}
$$

where $\theta_{i}, \theta_{j}$ are the angle of voltages at node $i$ and $j, x_{i j}$ is the reactance of branch $i j, P_{i j}$ is the active power on this branch. Since this method only deal with active power, for simplicity, the term 'power' in this paper refer to 'active power'.

The matrix form of (1) can be written as

$$
\boldsymbol{P}_{\text {line }}=\operatorname{diag}\left(\boldsymbol{B}_{\text {line }}\right) \boldsymbol{M}_{\text {red }}^{\mathrm{T}} \theta_{\text {red }}
$$

where $\boldsymbol{P}_{\text {line }}$ is the $b$ dimensioned column vector composed of branch power flows, $\boldsymbol{B}_{\text {line }}$ is the $b$ dimensioned column vector composed of branch susceptance, $\operatorname{diag}\left(\boldsymbol{B}_{\text {line }}\right)$ is the diagonally matrix of $\boldsymbol{B}_{\text {line }}, \boldsymbol{M}_{\text {red }}$ is the $((n-1) \times b)$ dimensioned reduced bus-branch incidence matrix deleting reference node, $\theta_{\text {red }}$ is the $(n-1)$ dimensioned reduced column vector of voltage angles of nodes deleting reference node.

$$
\text { From } \boldsymbol{B}_{\text {bus_red }} \theta_{\text {red }}=\boldsymbol{P}_{\text {bus_red }} \text { we would get: }
$$

$$
\boldsymbol{\theta}_{\text {red }}=\boldsymbol{X}_{\text {bus_red }} \boldsymbol{P}_{\text {bus_red }}
$$

where $\boldsymbol{B}_{\text {bus_red, }}, \boldsymbol{X}_{\text {bus_red }}$ are the $((n-1) \times(n-1))$ dimensioned reduced node sesceptance and reactance matrixes deleting reference node. $\boldsymbol{P}_{\text {bus_red }}$ is the $(n-1)$ dimensioned reduced node power injection column vector deleting reference node.

According to definition of GSDF, branch flows can be written as

$$
P_{\text {line }}=D_{G S D F_{-} \text {red }} P_{\text {bus_red }}
$$

where $\boldsymbol{D}_{\text {GSDF_ red }_{\text {r }}}=\operatorname{diag}\left(\boldsymbol{B}_{\text {line }}\right), \boldsymbol{M}_{\text {red }}{ }^{\mathrm{T}} \overline{\boldsymbol{X}}_{\text {bus_red }}$. Elements of matrix $D_{G S D F_{-}}$red are the traditional Generation Shift Distribution Factor (GSDF). Element of matrix $\boldsymbol{D}_{G S D F_{-} \text {red }}$ at row $i$ and column $j$ presents power flow change on branch $i$ when power injection at node $j$ is increased and at reference node is decreased by a unit power. With these factors, contribution of power injection at all nodes except reference node to the branch flows can be easily calculated. In fact, it can be regarded that change of power injection at reference node is balanced by reference node itself so it has no impact on the network power flow. Adding a column with all zero elements, the full-dimensioned distribution factors matrix $D_{G S D F}$, in which reference node is included, could be gotten.

If node injections corresponding to transactions are known, contribution of the transactions on branches can be calculated based on $D_{G S D F}$ :

$$
P_{\text {line,trade }}=D_{G S D F} P_{\text {trade }}, P_{\text {trade }} E_{(t)}=P_{\text {bus }}
$$

where $\boldsymbol{P}_{\text {trade }}=\left[\boldsymbol{P}_{\text {trade, } \mathbf{1}}, \boldsymbol{P}_{\text {trade, }, 2}, \cdots, \boldsymbol{P}_{\text {trade, }}\right]$ is the $(n \times t)$ dimensioned matrix of node power injections corresponding to transactions, $t$ is the number of transactions, $\boldsymbol{P}_{t r a d e, \mathbf{k}}$ is the node injection vector of transaction $k, \boldsymbol{E}_{(t)}$ is the $t$ dimensioned all-one column vector, $\boldsymbol{P}_{\text {line,trade }}$ is the $(b \times t)$ dimensioned matrix of contribution of transactions to branch power. $\boldsymbol{P}_{b u s}$ is the $n$ dimensioned node injection column vector, including reference node.

Having the usage price of each branches, the usage-based charge of transactions can be gotten:

$$
\begin{gathered}
T_{\text {trade }}=\gamma_{\text {ine_use }}{ }^{T} P_{\text {line,trade }} \\
=\gamma_{\text {ine_use }}{ }^{T} D_{G S D F} P_{\text {trade }}=\gamma_{\text {bus }} P_{\text {trade }}
\end{gathered}
$$

where $\boldsymbol{T}_{\text {trade }}$ is $t$ dimensioned row vector of transmission usage-based charge of transactions; $\chi_{\text {ine use }}$ is $b$ dimensioned column vector of prices of transmission usage-based charge, elements of which present the transmission usage-based tariff of unit power flow on each branch. $\gamma_{\text {wus }}$ is $n$ dimensioned column vector of prices of transmission usage charge of node power injections.

We can get from (6) that TUTs are determined by GSDFs and locations and magnitudes of node injections corresponding to transactions. Since GSDFs are only determined by network structure (topology and branch parameters) and have no relation with transactions, it is easy for market participants to estimate TUTs in advance. Since all transactions are balanced transactions, the result will not be 
affected by the selection of reference node. Since this method allocate TUT based on transactions but not on single generation or load and each transaction can be expressed as a node injection vector, this method is applicable to electricity markets with various trading mode such as bilateral, multilateral and pool trading modes, provided that all transactions are balanced transactions.

\section{ALLOCATION OF TUT AMONG PARTICIANTS IN POOL OR MÚLTILATERAL TRANSACTIONS}

Physical-flow based power flow tracing method can be employed for further distributing the charge obtained through GSDF based method described in the former section among the participants of each transaction concerned.

\section{A. Calculation steps}

First form $(n \times b)$ dimensioned bus-from-branch incidence matrix $\boldsymbol{M}_{\text {from }}$, which can be gotten by set all elements of incidence matrix $\boldsymbol{M}$ equaling to -1 to 0 , and the $(n \times b)$ dimensioned bus-to-branch incidence matrix $\boldsymbol{M}_{t o}$, which can be gotten by set all elements of incidence matrix $\boldsymbol{M}$ equaling to 1 to 0 . After that, for each transaction; TUTs of its participants can be calculated. The steps include:

a) to calculate the branch flow caused by this transaction according to its node injection vector;

$$
\boldsymbol{P}_{\text {line }, k}=\boldsymbol{D}_{\text {GSDF }} \boldsymbol{P}_{\text {trade, } k}
$$

where $\boldsymbol{P}_{\text {trade, } k}$ is the column vector of node injection of transaction $k, \boldsymbol{P}_{\text {line, },}$ is the column vector of branch flows caused by transaction $k$.

b) to calculate the column vector of node pass flow caused by transaction $k, \mathbf{P}_{\text {pass,k }}{ }^{[5]}$;

$$
\boldsymbol{P}_{p a s s, k}=\boldsymbol{M}_{\text {to }} \operatorname{diag}\left(\boldsymbol{P}_{\text {line, } k}\right) \boldsymbol{E}_{(b)}+\boldsymbol{P}_{\text {gen }, k}
$$

where $\boldsymbol{E}_{(b)}$ is the $b$ dimensioned column vector with all ones elements, $\boldsymbol{P}_{\text {gen, }, k}$ is the $n$ dimensioned column vector of node generation injection corresponding to transaction $k$.

c) to calculate the contribution of generations corresponding to transaction $k$ to branch flows using upstream tracing method;

$$
\begin{aligned}
& D_{\text {trace_gen,k}}=\operatorname{diag}\left(P_{\text {line }, k}\right) M_{\text {from }}{ }^{T}\left(\operatorname{diag}\left(P_{\text {pass }, k}\right)\right)^{-1} A_{u, k}{ }^{-1} \\
& \text { where } \\
& A_{u, k}=M_{\text {to }} \operatorname{diag}\left(P_{\text {line }, k}\right) M_{\text {from }}{ }^{T}\left(\operatorname{diag}\left(P_{p a s s, k}\right)\right)^{-1}+I
\end{aligned}
$$

$D_{\text {trace_gen,k }}$ is the matrix of contribution of generations corresponding to transaction $k$ to branch flows, $I$ is the $n$ dimensioned unit matrix.

d) to calculate contribution of loads corresponding to transaction $k$ to branch flows using down-stream tracing method;

$$
\begin{aligned}
& \mathbf{D}_{\text {trace_load,k }}=\operatorname{diag}\left(\mathbf{P}_{\text {line }, \mathbf{k}}\right) \mathbf{M}_{\mathrm{to}}{ }^{\mathrm{T}}\left(\operatorname{diag}\left(\mathbf{P}_{\mathrm{pass}, \mathrm{k}}\right)\right)^{-1} \mathbf{A}_{\mathrm{d}, \mathbf{k}}^{-1} \\
& A_{d, k}=M_{\text {from }} \operatorname{diag}\left(P_{\text {line }, k}\right) M_{\text {to }}{ }^{T}\left(\operatorname{diag}\left(P_{\text {pas }, k}\right)\right)^{-1}+I
\end{aligned}
$$

$D_{\text {trace_load, }}$ is the matrix of contribution of loads corresponding to transaction $k$ to branch flows.

e) to calculate the TUT of each participant in the transaction according to the pre-determined allocation ratio of total TUT between generations and loads.

Denote $a_{g}$ the percentage of total TUT allocated to generations, then $a_{l}=1-a_{g}$ is the percentage of total TUT allocated to loads

$$
\begin{aligned}
& T_{\text {use_gen, } k}=a_{g} P_{\text {price_line_use }}{ }^{T} D_{\text {trace_gen, } k} \operatorname{diag}\left(P_{\text {gen }}\right) \\
& T_{\text {use_load }, k}=a_{l} P_{\text {price_line_use }}{ }^{T} D_{\text {trace_load, } k} \operatorname{diag}\left(\boldsymbol{P}_{\text {load }}\right)
\end{aligned}
$$

$T_{\text {use_gen }, k}$ and $T_{\text {use_load }, k}$ are the vectors of TUTs of generations and loads corresponding to transaction $k$ respectively.

\section{B. Some special issues}

\section{1) Zero pass flow nodes}

Since each transaction may only refer to a few nodes, for some transactions, pass flows of some nodes may be zero, which will make inverse of some matrixes difficult. To solve this issue, the flowing approach can be used:

a) to delete elements in the node pass flow vector where the value is zero.

b) to delete the lines of the bus-branch incidence matrix corresponding to zero pass flow nodes.

c) to calculate the contribution matrixes of generations and loads to branch flows deleting zero pass flow nodes.

d) to add a zero column to the contribution matrixes of generations and loads to branch flows at the locations corresponding to each zero passing flow node.

e) To calculate the TUT of each participant.

\section{2) Counter flows}

In principle, power flow tracing method has no counter flow problem. But when it is synthesized with GSDF based method, it is possible that the power flows on some branches caused by a transaction may be different with the actual flow. In this circumstance, to assure the sum of the TUTs allocated to all participants of a transaction equals to total TUT of this transaction, it will be necessary to do some adjustment on the TUT prices of the branches.

For the problem that whether transactions causing counter flows should pay for using the network, many approaches have been proposed but neither have been widely accepted. There are many debates on this problem and we will not discuss it in this paper. From viewpoint of cooperation game theory, the transactions causing counter flows not only should not pay for the network usage, but also should get credit, so if direction of net flow on branch $i j$ caused by all the transactions is $i>j$, the TUT price of the branch for flows from $i$ to $j$ will be positive and that for flows form $j$ to $i$ will be negative. For a multilateral transaction, say, transaction $k$, if the flow on branch $i j$ caused by the transaction is from $j$ to $i$, the price of TUT of the branch for this transaction should be the opposite number of the initial price.

\section{DEAL WITH CROSS-REGION TRANSACTIONS}

Above discussion is limited in calculation of TUTs of transactions and participants in a single market. The proposed method is easy to be employed to interconnected multipleregional and hierarchical electricity markets. 
For example, in an interconnected power system, there are $m$ regional electricity markets. Branches inside each region belong to a separate TO (Transmission Owner) and all the tieline branches belong to an upper level TO. There are crossregional transactions among the regional electricity markets. The flowing method can be employed to calculate the TUTs:

a) Each TO submits (declare) TUT prices of its branches.

b) Firstly cross-regional transactions are dealt with. The TUT of each cross-region transaction is calculated with GSDF based method.

c) For cross-regional multilateral transactions, the total TUT should be allocated to different regions according to power flow tracing method, assuming that each TO presenting the corresponding region as the market participant.

d) For TUT of cross-region transactions allocated to each region, it is the responsibility of each region to allocate it to the corresponding generations and loads.

e) Then the intra-area transactions are dealt with. If there are bilateral and multilateral transactions, the TUTs are calculated through the proposed GSDF based method and power flow tracing method. If there are no bilateral and multilateral transactions, that is, there is only a pool transaction, the power flow tracing based method is directly used to calculate the TUT of the participants.

There is no difference between the calculation of the TUT of intra-area branch and tie-line branches.

In practice, transmission users usually only pay to the native TO and then the TOs clear each other for crossregional transactions.

\section{Digital EXAMPLE}

The IEEE-30 network is used to illustrate the proposed method. There are 30 buses and 41 branches in the system. Suppose that there are three regions: region 1, region 2 and region 3 . Buses 1-8 belong to region 1; buses $9-20$ belong to region 2 and buses $21-30$ belong to region 3 . Also suppose that the 41 branches belong to 4 TOs. Branches inside each area belong to a separate TO (TO1, TO2 and TO3), and all tie-lines belong to another TO, TO4. For simplicity, suppose that all branches have the same TUT price, that is, $1 \$ / \mathrm{pu}$.

There are 6 balanced transactions:

- Transaction 1: the pool transaction in region 1;

- Transaction 2: the pool transaction in region 2;

- Transaction 3: the pool transaction in region 3;

- Transaction 4: the cross-region transaction between area 1 and area 2 . The source buses are bus 1 and bus 2 in region 1 and the sink buses are bus 10 , bus 12 and bus 14 in region 2 . The quantities of node injections are listed in table 1 (a).

- Transaction 5: The cross-region transaction between region 1 and region 3 . The source buses are bus 2 and bus 5 in region 1 and the sink buses are bus 21, bus 23 and bus 24 in region 3 . The quantities of node injections are listed in table 1 (b).
- Transactions 6: The bilateral transaction in region 1 from bus 1 to bus 5 . The quantity of the transaction is $0.3 \mathrm{pu}$.

Table 2 lists the TUT of each transaction should paid to each of the four TOs. Negative TUTs in the table means that the corresponding transaction results in counter flows in some branches. Of cause, the counter flow problem can be handled with other approaches.

Table 1 lists the TUTs of participants in the two crossregion transactions. The coefficient $a_{g}$ equals to 0.3 , that is, $30 \%$ of the total transmission usage-based tariff is allocated to generations and the remaining $70 \%$ is allocated to loads. Comparing results of table 1 and table 2, we can get that the sum of TUTs allocated to all participants in each transaction well matches the total TUTs allocated to the corresponding transaction.

Based on the data in table 2, the TUTs, which should be paid by the transmission users (include generations and loads) in each region to each TO, could be calculated (see Table 3). For example, the TUTs should be paid by transmission users in region 1 are the sum of TUTs of transaction 1 and transaction 6 and the TUTs of the corresponding buses of transaction 4 and transaction5. The numbers in the 2-4 columns are TUTs paid to TO1, TO2 and TO3 by transmission users in different regions. The numbers in the fifth column are the total TUTs paid to the TOs, and the numbers in the last line are the total TUTs paid by transmission users in different areas. The numbers in the last column are the settlement among different regions, which mean that TO1, TO2 and TO3 all should pay part of TUTs they received to TO4.

TABLE 1

DATA OF CROSS-REGION TRADES

\begin{tabular}{|c|c|c|c|c|c|}
\hline \multicolumn{3}{|c|}{ (a) Trade 4 } & \multicolumn{3}{c|}{ (b)Trade 5 } \\
\hline No & $\mathrm{P}$ & $\begin{array}{c}\text { ToU } \\
\left(\mathrm{a}_{\mathrm{g}}=0.3\right)\end{array}$ & No & $\mathrm{P}$ & $\begin{array}{c}\text { ToU. } \\
\left(\mathrm{a}_{\mathrm{g}}=0.3\right)\end{array}$ \\
\hline 1 & 0.088 & 0.085 & 2 & 0.1 & 0.1264 \\
\hline 2 & 0.1 & 0.0632 & 5 & 0.159 & 0.0864 \\
\hline 10 & -0.058 & 0.1053 & 21 & -0.175 & 0.3016 \\
\hline 12 & -0.1 & 0.1752 & 23 & -0.032 & 0.0869 \\
\hline 14 & -0.03 & 0.0652 & 24 & -0.052 & 0.1081 \\
\hline sum & 0 & 0.4939 & 总 & 0 & 0.7094 \\
\hline
\end{tabular}

TABLE 2

TRANSMISSION USAGE-BASED TARIFF OF TRADES

\begin{tabular}{|c|c|c|c|c|c|}
\hline trade & TO1 & TO2 & TO3 & TO4 & sum \\
\hline 1 & 1.5946 & 0.0074 & -0.0123 & 0.0318 & 1.6215 \\
\hline 2 & -0.0079 & 1.1081 & 0.0502 & -0.0149 & 1.1355 \\
\hline 3 & -0.0097 & 0.0172 & 0.2439 & 0.0287 & 0.2801 \\
\hline 4 & 0.2426 & 0.0245 & 0.0611 & 0.1657 & 0.4939 \\
\hline 5 & -0.0887 & 0.1803 & 0.1529 & 0.4650 & 0.7095 \\
\hline 6 & 0.9520 & 0.0034 & -0.0051 & 0.0015 & 0.9518 \\
\hline sum & 2.6829 & 1.3409 & 0.4907 & 0.6778 & 5.1923 \\
\hline
\end{tabular}

TABLE 3

TRANSMISSION USAGE-BASED TARIFF OF GENERATORS AND LOADS OF EACH REGION PAID TO TRANSMISSION OWNERS 


\begin{tabular}{|c|c|c|c|c|c|}
\hline TO & Area1 & Area 2 & Area 3 & Sum in & Net in \\
\hline 1 & 2.5928 & 0.1619 & -0.0718 & $2.6829(1)$ & $-0.2514 \mathrm{~A}$ \\
\hline 2 & 0.0722 & 1.1253 & 0.1434 & 1.3409 (2) & $-0.1404 \mathrm{~B}$ \\
\hline 3 & 0.0468 & 0.0930 & 0.3509 & $0.4907(3)$ & $-0.2860 \mathrm{C}$ \\
\hline 4 & 0.2225 & 0.1011 & 0.3542 & $0.6778(4)$ & $0.6778 \mathrm{D}$ \\
\hline sum & 2.9343 & $\mathrm{~b}_{1.4813}$ & 0.7767 & $\mathrm{~d}_{5.1923}$ & ${ }^{e} 0$ \\
\hline
\end{tabular}

\section{CONCLUSIONS}

Based on GSDF based method and power flow tracing method, this paper presented an integrated approach for allocating transmission usage-based tariff among generators and loads. The proposed method is easy to implement and is applicable to electricity markets with various trading modes such as bilateral, multilateral and pool trading modes, and to interconnected multiple-regional and hierarchical electricity markets as well.

\section{APPENDIX}

TABLE 4

THE BUS DATA OF IEEE-30 NETWORK

\begin{tabular}{|c|c|c|c|c|c|}
\hline No & $\mathbf{P}_{\text {gen }}$ & $P_{\text {load }}$ & No & $P_{\text {gen }}$ & $P_{\text {load }}$ \\
\hline 1 & 0.734 & 0.0 & 16 & 0.000 & 0.035 \\
\hline 2 & 0.800 & 0.217 & 17 & 0.000 & 0.090 \\
\hline 3 & 0.000 & 0.024 & 18 & 0.000 & 0.032 \\
\hline 4 & 0.000 & 0.076 & 19 & 0.000 & 0.095 \\
\hline 5 & 0.500 & 0.942 & 20 & 0.000 & 0.022 \\
\hline 6 & 0.000 & 0.0 & 21 & 0.000 & 0.175 \\
\hline 7 & 0.000 & 0.228 & 22 & 0.000 & 0.0 \\
\hline 8 & 0.200 & 0.300 & 23 & 0.000 & 0.032 \\
\hline 9 & 0.000 & 0.0 & 24 & 0.000 & 0.087 \\
\hline 10 & 0.000 & 0.058 & 25 & 0.000 & 0.0 \\
\hline 11 & 0.200 & 0.0 & 26 & 0.000 & 0.035 \\
\hline 12 & 0.000 & 0.112 & 27 & 0.200 & 0.0 \\
\hline 13 & 0.200 & 0.0 & 28 & 0.000 & 0.0 \\
\hline 14 & 0.000 & 0.062 & 29 & 0.000 & 0.024 \\
\hline 15 & 0.000 & 0.082 & 30 & 0.000 & 0.106 \\
\hline
\end{tabular}

\section{REFERENCES}

[1] Ng W Y. Generalized generation distribution factors for power system security evaluation. IEEE Trans. on Power Apparatus and Systems, 1981, 100(3): 1001-1005.

[2] Shirmohammadi D, Gribik P R, Law E T K, et al. Evaluation of transmission network capacity use for wheeling transactions. IEEE Trans. on Power Systems, 1989, 4(4): 1405-1413.

[3] Rudnick H, Palma R, Fernandez J E. Marginal pricing and supplement cost allocation in transmission open access. IEEE Trans. on Power Systems, 1995, 10(2): 1125-1142.

[4] Rudnick H, Soto $M$ and Palma R. Use of system approaches for transmission open access pricing. Electrical Power and Energy Systems 21(1999): 125-135.

[5] Bialek J. Tracing the flow of electricity. IEE Proc.-Gener. Transm.Distrib., 1996, 143(4): 313-321.

[6] Bialek J. Topological generation and load distribution factors for supplement charge allocation in transmission open access. IEEE Trans. on Power Systems, 1997, 12(3): 1185-1193.

[7] Bialek J. Allocation of transmission supplementary charge to real and reactive loads. IEEE Trans. on Power systems, 1998, 13(3): 749-754.

\section{BIOGRAPHIES}

Zhaoxia Jing was born in Henan, China, in 1975. She received her B.E. degree in electrical engineering from the Huazhong University of Science and Technology(HUST) in 1997 and now she is pursuing her Ph.D. in the same university. Her major area of work is electricity market.

Xianzhong Duan was born in Hunan, China, in 1968. He graduated from HUST in 1987, and received his Ph.D. degree in electrical engineering at the same university in 1992. Since then he has been working at HUST, now he is a full professor. His research interests include power system analysis, electricity market and informational of power systems.

Yixin Ni (Senior Member', IEEE) received her B. Eng., M. Eng., and Dr. Eng. all from Electrical Engineering Department, Tsinghua University, P.R. China in 1968, 1981 and 1983 respectively. Her research interests are in power system modeling, simulation, stability and control, and power electronics applications in power systems. She was a professor of Tsinghua University and is now with the University of Hong Kong. She is a senior member of CSEE and Fellow of HKIE.

Fushuan Wen received his BE and ME degrees from Tianjin University, China, in 1985 and 1988, respectively, and $\mathrm{PhD}$ from Zhejiang University, China, in 1991, all in electrical engineering. He joined the faculty of Zhejiang Unversity, China, in 1991, and has been professor there since 1997. He held a visiting position in the National University of Singapore from 1995 to 1997 , and was a research fellow in the Hong Kong Polytechnic University from July 1998 to February 2001. He is now a research assistant professor in the University of Hong Kong. His current research interests are in power industry restructuring and artificial intelligence applications in power systems.

Felix F. Wu (Fellow, IEEE) received his Ph. D. from University of California at Berkeley (UCB). He is now a chair professor of electrical engineering at the University of Hong Kong. Prior to that he was a professor and vice chairman of Dept. of EECS, UCB. His research interests are in power industry restructuring, power system investment planning, design of modern control centers, distribution automation, distributed processing etc. 